\title{
MITTEILUNGEN
}

\section{Parlamentarisierung in der EU - Chancen und Grenzen. Eine Veranstaltung der DVParl am 9. April 2014 in Berlin}

Im Vorfeld der Abendveranstaltung zum Thema „Parlamentarisierung in der EU - Chancen und Grenzen" fand am 9. April 2014 die Jahresversammlung der Deutschen Vereinigung für Parlamentsfragen (DVParl) in Berlin statt, auf der ein neuer Vorstand zu wählen war: Den Vorsitz wird künftig die Bundestagsabgeordnete Eva Högl innehaben. Die stellvertretende Vorsitzende der SPD-Bundestagsfraktion löst Brigitte Zypries ab, die seit Dezember 2013 Parlamentarische Staatssekretärin beim Bundesminister für Wirtschaft und Energie ist. Zu Stellvertretern wurden Heinrich Oberreuter (Universität Passau und Direktor der Akademie für Politische Bildung in Tutzing a.D.), Georg Paul Hefty (Journalist) und Patrick Sensburg (MdB und Fachhochschule für öffentliche Verwaltung in Münster) gewählt.

Im Anschluss an die Mitgliederversammlung beleuchtete die DVParl die Parlamentarisierung in der Europäischen Union - ein Dauerthema seit der ersten Direktwahl zum Europäischen Parlament (EP) 1979. Im Mittelpunkt stand die Diskussion um das europäische Entscheidungs-, Regierungs- und Repräsentationssystem, das zwar grundsätzlich dieselben Funktionen zu erfüllen hat wie in jedem nationalen Regierungssystem auch, nur auf eine andere Weise. „Die abziehbildhafte Übertragung nationaler Institutionen auf das europäische Systemgefüge ist immer ein Irrtum gewesen“, wie Oberreuter als Moderator der Veranstalter gleich zu Beginn betonte. Aus drei unterschiedlichen Blickwinkeln betrachteten Luc Frieden (ehemaliger Finanzminister Luxemburgs), Andreas Maurer (Institut für Politikwissenschaft an der Universität Innsbruck) und Frank Schorkopf (Institut für Völkerrecht und Europarecht, Abteilung Europarecht an der Georg-August-Universität Göttingen) in ihren Vorträgen das Thema des Abends. ${ }^{1}$

Zum Auftakt hielt Luc Frieden ein sachliches Plädoyer, in dem er den Zuhörern den Weg von der Symbolik zu den Institutionen, von den Emotionen zur Rationalität und von der Intergouvernementalität zurück zu europäisch integrierten Entscheidungswegen wies. Er machte darauf aufmerksam, dass nicht nur eine europäische Öffentlichkeit notwendig ist, in der der Mehrwert Europas diskutiert wird; vielmehr müsse die Forderung, mehr Kompetenzen an die europäischen Institutionen abzugeben, angesichts der zukünftigen Herausforderungen auch mit legitimatorischen Bemühungen begleitet werden.

Aus seiner langjährigen Erfahrung als Minister komme er jedoch auch zu der Erkenntnis, dass nicht alle Mitgliedstaaten gleich zu behandeln seien. „Deshalb glaube ich, dass eine der Lösungen sein kann, dass wir Sockelelemente haben, wo einige mitmachen, wobei an den nächsten Stufen andere nicht teilnehmen“, wie beispielsweise bereits beim Schengener Abkommen und der Europäischen Wirtschafts- und Währungsunion. „Am Ende des

1 Vgl. auch Sandra Ketterer, Macht und Ohnmacht der Europa-Abgeordneten, in: Das Parlament vom 14. April 2014, Kehrseite. 
Tages“, so Friedens Prognose, „wird Europa eine föderale Struktur sein, selbst wenn diese in der Zeit verschoben wird.“

Im Mittelpunkt von Andreas Maurers Vortrag stand das Europäische Parlament. Seine Ausführungen belegten, dass es in der EU nicht nur eine andere Zuweisung von Funktionen auf die Institutionen, sondern auch eine andere Politische Kultur gibt. Da aus nationalstaatlicher Perspektive vieles widersprüchlich scheint, ist es nicht einfach, Strategien zu entwickeln, die die Position des Europäischen Parlaments im Institutionengefüge wie auch nach außen im Legitimationsprozess stärkten. Maurer warf im Laufe des Abends einen Blick darauf, in welchem Maße das EP an Entscheidungen beteiligt ist und kam zu dem Schluss, dass viele Rechtsetzungsakte zustimmungsbedürftig seien - das Parlament sich also damit beschäftigen muss. Im Verlauf des europäischen Einigungsprozesses ist die Verantwortung der Abgeordneten des EP stetig gestiegen.

Zugleich können positiv scheinende Entwicklungen auch kontraproduktiv sein: Der Vertrag von Lissabon schreibt vor, dass das Europäische Parlament den (vom Europäischen Rat) vorgeschlagenen Präsidenten der Europäischen Kommission wählt. Da der Rat bei seinem Vorschlag das Ergebnis der Europawahl berücksichtigen muss, haben die großen Parteien auf europäischer Ebene vor der Wahl 2014 erstmals Kandidaten für das Amt des Kommissionspräsidenten aufgestellt. So zeigt einerseits der Wahlkampf zum EP den Versuch der Parteien, stärker zu politisieren und personalisieren. Damit verbunden ist die Hoffnung, dass sich durch das Aufstellen von Spitzenkandidaten nicht nur die Mobilisierung der Wähler erhöht, sondern auch die Kommunikations- und Repräsentationsfunktion sowie die generelle Wahrnehmung des Parlaments als politischer Akteur gestärkt wird.

Auf der anderen Seite zeigt die Empirie, dass ein politisiertes, polarisiertes Parlament eher gehemmt ist. So machte Maurer deutlich, „dass der Ministerrat schwache und parteipolitisch eindeutige Mehrheiten zu seinen Gunsten nutzen wird und das Parlament immer wieder destabilisiert (...), und das würde bei der Realisierung eines parteipolitisierten Parlaments mit Mehrheit und Opposition noch viel stärker zum Vorschein kommen“. Auch würden die Trilogverhandlungen - mit denen Parlament, Rat und Kommission in kleiner Runde eine Einigung bei Gesetzesmaßnahmen erzielen wollen - noch stärker genutzt, um die Abgeordneten gegeneinander auszuspielen. Hier werde das Parlament schon jetzt oft „über den Tisch gezogen“, so dass Maurer dafür plädierte, das Verfahren auf Routinemaßnahmen zu beschränken.

Der dritte Redner, Frank Schorkopf, brachte gleich am Anfang seine Position auf den Punkt: Die EU sei für ihn nicht das perfektionierte Modell einer supranationalen Demokratie, bei dem es um die Frage gehe, wie die Kompetenzen des Parlaments weiter gestärkt werden können. Vielmehr sei „die Gemeinschaftsmethode an eine Leistungsgrenze gestoßen“, weshalb aus seiner Perspektive zuletzt vermehrt auf die Unionsmethode, also das Intergouvernementale, zurückgegriffen worden sei. Eine Zusammenarbeit einzelner Staaten innerhalb der EU halte er „keineswegs für die zweitbeste Lösung“. So könne auch er sich beispielsweise vorstellen, dass es eine Europäische Gemeinschaft gebe, die in unterschiedlichen Stufen miteinander verbunden sei.

Auch sei - im Gegensatz zu den Mitgliedstaaten - die Europäische Union für ihn weiterhin „kein politischer Primärraum“. So hätte unter anderem die Finanzkrise gezeigt, dass sich die Erwartungen der Bürger zuerst an die Nationalstaaten und nicht an die EU richten. Insgesamt reiche der Unionsbürgerstatus und der politische Wille für Europa nicht 
aus, „dauerhaft Mehrheitsentscheidungen im Europäischen Parlament im Hinblick auf die strukturelle Minderheit zu tragen “. Aus diesem Grund sind für Schorkopf komplementäre Integrationsmethoden im Sinne einer intergouvernementalen Integration ein vielversprechenderer Weg.

Die anschließende Diskussion vertiefte die Frage, wie das EP zu stärken sei. Unter reger Beteiligung der Bundestagsabgeordneten Elisabeth Winkelmeier-Becker und Katarina Barlay sowie den ehemaligen Parlamentariern Wolfgang Wieland und Nils Diederich erörterten die Referenten, welche bestehenden Kompetenzen im Zuge der Rahmenabkommen mit der Kommission oder auch der Zentralbank besser genutzt und welche - beispielsweise bei den Anhörungsrechten - zunächst ohne vertragliche Änderungen zusätzlich übertragen werden könnten. Außerdem ließe sich die Wahrnehmung des Europäischen Parlaments verbessern, indem sich die Abgeordneten nicht mehr darauf verließen, dass politische Inhalte über das Generalsekretariat und die Generaldirektion Presse und Kommunikation vermittelt werden, sondern dies über eigene Pressedienste der einzelnen Fraktionen geschieht.

Der Abend bot unterschiedliche Positionen: ein flammendes Plädoyer für ein gestärktes Europäisches Parlament verknüpft mit einer aktivierten europäischen Öffentlichkeit, aber auch die Gegenposition - ein politischer Primärraum sei die Europäische Union nicht, und mit der Legitimationskraft des Europäischen Parlaments auf Basis des Mehrheitsprinzips sei es nicht so weit her. So blieben die Zuhörer auf den ersten Blick ein wenig ratlos zurück. Bei genauer Betrachtung hat die Veranstaltung der Deutschen Vereinigung für Parlamentsfragen allerdings wieder einmal gezeigt, dass es sich lohnt, sich mit unterschiedlichen Positionen auseinanderzusetzen - besonders wenn sie das gemeinsame Interesse haben, Entscheidungsstrukturen und -verfahren in der Europäischen Union zu finden, die legitimatorisch akzeptabel sind und effiziente Problemlösungen erlauben. 